TRANSACTIONS OF THE

AMERICAN MATHEMATICAL SOCIETY

Volume 352, Number 6, Pages 2491-2505

S 0002-9947(99)02400-9

Article electronically published on April 7, 1999

\title{
ON BETTER-QUASI-ORDERING COUNTABLE SERIES-PARALLEL ORDERS
}

\author{
STÉPHAN THOMASSÉ
}

\begin{abstract}
We prove that any infinite sequence of countable series-parallel orders contains an increasing (with respect to embedding) infinite subsequence. This result generalizes Laver's and Corominas' theorems concerning betterquasi-order of the classes of countable chains and trees.
\end{abstract}

Let $\mathcal{C}$ be a class of structures and $\leq$ an order on $\mathcal{C}$. This class is well-quasiordered with respect to $\leq$ if for any infinite sequence $C_{1}, C_{2}, \ldots, C_{k}, \ldots$ in $\mathcal{C}$, there exist $i<j$ such that $C_{i} \leq C_{j}$. An equivalent definition is: any subset of $\mathcal{C}$ has finitely many minimal elements with respect to $\leq$. We are mainly concerned here with binary relations, the order $\leq$ being the embedding order. Thus, $R \leq R^{\prime}$ when the relation $R$ is embedded in the relation $R^{\prime}$ (in other words, $R$ is an induced relation of $R^{\prime}$ ). One of the very first results concerning well-quasi-order is Higman's theorem ([5]): the class of finite linear orderings (or chains) labeled by a finite set is well-quasi-ordered with respect to embedding. More precisely, the objects of the class are finite chains whose elements are labeled by a finite set, and embedding means order-preserving and label-preserving injection. For example aabcc embeds into $a b c a b c a b c$ but not into cbacbacba. This result was extended by Kruskal to the class of finite trees [8]. Then Nash-Williams introduced two fundamental tools of the theory: the 'minimal bad sequence' which shortened greatly the proofs concerning well-quasi-order (wqo), and the 'better-quasi-order' (bqo), a strengthening of wqo, which provides a tool to deal with countable structures [11. Indeed, wqo is no longer an appropriate tool in the infinite case, since one can construct, from Rado's counterexample [13], an infinite set of pairwise incomparable countable subsets of a wqo. Laver proved in [9] that the class of countable chains is better-quasi-ordered (and thus well-quasi-ordered) with respect to embedding. Later, Corominas [1] extended the result of Kruskal to countable trees: the class of countable trees labeled by a better-quasi-order is better-quasi-ordered with respect to embedding. The proof is in two parts, one devoted to the construction of countable trees, the other concerned with the preservation of better-quasi-order under certain operations. This latter aspect can be found in Milner [10] and Pouzet [12]. In this paper, Pouzet poses the problem of another class of orders, the $N$-free or series-parallel orders. This class contains the class of trees, and Pouzet was able to prove the wqo

Received by the editors October 4, 1995 and, in revised form, January 6, 1998.

1991 Mathematics Subject Classification. Primary 05C20; Secondary 05C05,08A65,05C75.

Key words and phrases. Better-quasi-order, interval, series-parallel graphs, series-parallel orders, structured trees, well-quasi-order.

This work was supported in part by the DIMANET program, Human Capital and Mobility (HCM) Contract No. ERBCHRXCT 940429. 
character of the class of finite series-parallel orders. He conjectured also that the class of countable series-parallel orders is better-quasi-ordered. We settle this question affirmatively in this paper. The proof is in two parts. First, we translate this problem into a structured tree problem, by mean of a decomposition tree based on the notion of interval, a method which can be found in Kelly's survey 7]. Second, we extend Corominas' theorem to the class of structured trees. We conclude the paper with a discussion of the challenging compactness conjecture of Pouzet.

\section{The ClASS OF SERIES-PARALLEL GRAPHS}

Definition 1. A binary relation is a pair $R=(V, E)$ where $V$ is a set and $E$ a subset of $V \times V$. The elements of $V$ are the vertices of $R$; those of $E$ are the edges of $R$. A binary relation $R=(V, E)$ is embedded into another binary relation $S=(W, F)$ (or $S$ embeds $R$ ) if there is an injective mapping $f$ from $V$ into $W$ such that $(x, y) \in E$ if and only if $(f(x), f(y)) \in F$. If $f$ is a bijective mapping, then $R$ is isomorphic to $S$. For $Y$ a subset of $V, R(Y)=(Y,(Y \times Y) \cap E)$ is the induced subrelation on $Y$. Hence, the relation $R$ is embedded into $S$ if and only if $R$ is isomorphic to an induced subrelation of $S$. If both $R$ embeds $S$ and $S$ embeds $R$, then $R$ is equimorphic to $S$. A subset $Y$ of $V$ is a copy of $R$ if $R(Y)$ is isomorphic to $R$.

A graph $G=(V, E)$ is an irreflexive and symmetric binary relation. If $(x, y) \in E$, then $x$ is adjacent to $y$. If $x$ is adjacent (resp. not adjacent) to $y$ for any $x \neq y$, then $G$ is a complete graph (resp. an empty graph). The complement of $G=(V, E)$ is the graph $G^{c}=(V,(V \times V) \backslash(E \cup\{(x, x): x \in V\}))$. Let $G=(V, E)$ and $H=(W, F)$ be two graphs such that $V \cap W=\emptyset$. The graph $G \cup H=(V \cup W, E \cup F)$ is the union of $G$ and $H$. We refer to the union between graphs as the parallel operation. The graph $G \cup H$ is a parallel graph provided that $G$ and $H$ both have at least one vertex. The graph $G+H=(V \cup W, E \cup F \cup(V \times W) \cup(W \times V))$ is the sum of $G$ and $H$. We refer to the sum between graphs as the series operation. The graph $G+H$ is a series graph provided that $G$ and $H$ both have at least one vertex. The smallest class of graphs which contains the one-element graph and which is closed under parallel and series operations is called the class of finite series-parallel graphs.

A path of $G=(V, E)$ is a sequence of distinct vertices $v_{0}, v_{1}, \ldots, v_{n}$, such that $v_{i}$ is adjacent to $v_{i+1}$ for all $i \in\{0, \ldots, n-1\}$; this path connects $v_{0}$ and $v_{n}$. A graph is connected if any two of its vertices are connected by a path. A maximal connected induced subgraph is a connected component. We denote by $N$ the graph with vertex set $\{1,2,3,4\}$ and edge set $\{(1,2),(2,3),(3,4)\}$. A graph is $N$-free if it does not embed $N$.

Lemma 1 (Gallai [4). The class of finite series-parallel graphs is the class of finite $N$-free graphs.

Proof. Since the graph $N$ is not series-parallel, every series-parallel graph is $N$-free. We prove the converse by induction on $n$. Let $G=(V, E)$ be a $N$-free graph. If $G$ is either series or parallel, the result holds by the induction hypothesis. Suppose, by way of contradiction, that both $G$ and $G^{c}$ are connected. Let $x$ be a vertex of $G$. By the induction hypothesis $G-x$ is a series-parallel graph. Free to consider $G^{c}$ instead of $G$ (since $N^{c}$ is isomorphic to $N$ ), we may suppose without loss of generality that $G-x=G_{1} \cup G_{2}$, where $G_{1}$ and $G_{2}$ are nonempty. Denote by $V_{1}$ and $V_{2}$ the vertex sets of $G_{1}$ and $G_{2}$. Let $A_{1}=\left\{y \in V_{1}:(y, x) \in G\right\}, B_{1}=\left\{y \in V_{1}:(y, x) \notin G\right\}$, $A_{2}=\left\{y \in V_{2}:(y, x) \in G\right\}$ and $B_{2}=\left\{y \in V_{2}:(y, x) \notin G\right\}$. Since $G$ is connected, 
$A_{1}$ and $A_{2}$ are nonempty. Moreover $x$ is not adjacent in $G$ to all other vertices, so $B_{1} \cup B_{2} \neq \emptyset$. Suppose without loss of generality that $B_{1} \neq \emptyset$. There exists an edge $(u, v) \in E$ such that $u \in B_{1}$ and $v \in A_{1}$ (otherwise $G$ is not connected). Now consider a vertex $w \in A_{2}$. The induced subgraph $G(\{u, v, x, w\})$ is isomorphic to $N$, a contradiction.

The above lemma does not extend to the infinite case: there exist infinite $N$-free graphs $G$ such that both $G$ and $G^{c}$ are connected, for instance, the graph on the natural numbers with $2 n$ adjacent to $k$ for any $n$ and $k$ such that $k<2 n$. From now on, a series-parallel graph is an $N$-free graph.

\section{WELL-QUASI-ORDER OF FINITE SERIES-PARALLEL GRAPHS}

Definition 2. An order $P=(V, E)$ is a reflexive, antisymmetric and transitive binary relation. For an order $P$, we write $x \leq_{P} y$ (or $x \leq y$ when there is no ambiguity) instead of $(x, y) \in E$. If $x \leq y$ or $y \leq x$, then $x$ and $y$ are comparable (otherwise they are incomparable). The graph $G_{c}(P)=\left(V, E^{\prime}\right)$ such that $(x, y) \in E^{\prime}$ if $x$ is comparable to $y$ in $P$ is the comparability graph of $P$. An order such that any two of its vertices are comparable (resp. incomparable) is a chain (resp. an antichain). The connected components of $P$ are the connected components of $G_{c}(P)$. A subset $S$ of $V$ is a final section of $P$ (resp. an initial section of $P$ ) if for any $x \in S$ and $y \geq x$ (resp. $x \in S$ and $y \leq x$ ), $y$ also belongs to $S$. Let $W$ be a subset of $V$. The final (resp. initial) section generated by $W$ is the smallest final (resp. initial) section which contains $W$. For $x \in V$, the final section generated by $\{x\}$ is denoted by $V^{x}$, the initial section by $V_{x}$. We also write $P^{x}$ and $P_{x}$ instead of $P\left(V^{x}\right)$ and $P\left(V_{x}\right)$, respectively. Let $P=(V, E)$ and $Q=(V, F)$ be two orders defined on the same vertex set; $Q$ is an extension of $P$ if $E \subseteq F$.

Let $Q$ be an order and $R$ a relation; a $Q$-label of $R$ is a mapping $l$ from the set of vertices of $R$ into $Q$. We say that $(R, l)$ is a $Q$-labeled relation, where $Q$ is the range of $l$. Let $\left(R_{1}, l_{1}\right)$ and $\left(R_{2}, l_{2}\right)$ be two $Q$-labeled relations; $f$ is a $Q$ embedding from $\left(R_{1}, l_{1}\right)$ into $\left(R_{2}, l_{2}\right)$ if $f$ is an embedding from $R_{1}$ into $R_{2}$ and $l_{1}(x) \leq l_{2}(f(x))$ for any vertex $x$ of $R_{1}$. In other words, $\left(R_{2}, l_{2}\right) l$-embeds $\left(R_{1}, l_{1}\right)$. We permit multiple labeling; that is, precisely, we identify $Q_{1}$-labeled $Q_{2}$-labeled relations and $\left(Q_{1} \times Q_{2}\right)$-labeled relations.

A forest is an order $F$ such that $F^{x}$ is a chain for any vertex $x$ of $F$. A tree is a forest $T=(V, E)$ in which $V^{x} \cap V^{y} \neq \emptyset$ for all vertices $x, y$ of $T$. Since we want to represent inclusion, our definition of trees is the opposite of the usual one. Let $x$ be a vertex of $T$; a branch of $x$ is an induced, maximal (under inclusion) subtree of $T_{x}-x$. A tree is a binary tree if each of its vertices has at most two branches. When it exists, the minimum vertex (with respect to $T$ ) of $V_{x} \cap V_{y}$ is the supremum of $x$ and $y$; we denote it by $x \vee y$. A tree is well-branched if any two of its vertices have a supremum. Let $T$ and $T^{\prime}$ be well-branched trees, $f$ is a $\vee$-embedding from $T$ into $T^{\prime}$ if $f$ is an embedding from $T$ into $T^{\prime}$ and $f(x \vee y)=f(x) \vee f(y)$ for any vertices $x$ and $y$ in $T$. If moreover $T$ and $T^{\prime}$ are $Q$-labeled trees, a $Q$ - $\vee$-embedding from $T$ into $T^{\prime}$ is both a $Q$-embedding and a $\vee$-embedding.

The order $A_{3}$ is the antichain on the vertex set $\{0,1,2\}, A_{2}$ the antichain on the vertex set $\{0,1\}$. We will often need $A_{2}$ and $A_{3}$ in our labelings. We denote by $\mathcal{T}$ the class of $A_{3}$-labeled, binary trees such that minimal elements, and only these ones, are labeled by 0 . The subclass of finite trees of $\mathcal{T}$ is denoted by $\mathcal{T}_{F}$. To any labeled tree $(T, l)$ of $\mathcal{T}$, we associate a graph $S P((T, l))$ whose vertex set $M$ is the 
set of minimal elements of $T$ and whose edge set is $\{(x, y): l(x \vee y)=1\}$. Such a graph is series-parallel since $N$ cannot occur as an induced subgraph of $S P((T, l))$. Moreover, let $(T, l)$ and $\left(T^{\prime}, l^{\prime}\right)$ be two elements of $\mathcal{T}$. If $f$ is a $A_{3}$-V-embedding from $(T, l)$ into $\left(T^{\prime}, l^{\prime}\right)$, then the restriction of $f$ to the minimal elements of $(T, l)$ is an embedding from $S P((T, l))$ into $S P\left(\left(T^{\prime}, l^{\prime}\right)\right)$. This construction may also be applied to a labeled tree $\left((T, l), l^{\prime}\right)$ where $(T, l) \in \mathcal{T}$ and $l^{\prime}$ is an arbitrary label of $T$, in which case $S P\left(\left((T, l), l^{\prime}\right)\right)$ is the labeled graph $\left(S P((T, l)), l^{\prime \prime}\right)$ where $l^{\prime \prime}$ is the restriction of $l^{\prime}$ to the minimal elements of $T$.

A quasi-order $Q=(V, E)$ is a reflexive and transitive binary relation. A sequence $\left\{q_{i}\right\}_{i \in \omega}$ of elements of $P$ is good if there are $i<j$ such that $q_{i} \leq q_{j}$; otherwise the sequence is bad. A quasi-order $Q$ is a well-quasi-order (wqo) if any infinite sequence of elements of $Q$ is good. It follows from Ramsey's theorem that any infinite sequence of elements of a wqo contains an infinite increasing subsequence. An extension of a well-quasi-order is a well-quasi-order.

Let $\mathcal{C}$ be a class of relations and $Q$ a quasi-order. We denote by $Q^{\mathcal{C}}$ the class of $Q$-labeled relations of $\mathcal{C}$. Note that $\mathcal{C}$ may already be a class of $Q^{\prime}$-labeled relations, in which case $Q^{\mathcal{C}}$ is a class of $Q$-labeled $Q^{\prime}$-labeled relations. In order to choose an embedding order on $\mathcal{C}$, we systematically endow $\mathcal{C}$ with the strongest one; for instance if $\mathcal{C}$ is a class of $Q$-labeled well-branched trees, we order it by $Q$-V-embedding. In this sense, the embedding order on the class $\mathcal{C}$ is canonical, and we denote it by $\leq_{\mathcal{C}}$. Since isomorphism is no longer equivalent to equimorphism when we deal with infinite structures, $\left(\mathcal{C}, \leq_{\mathcal{C}}\right)$ is a quasi-order. The class $\mathcal{C}$ is wellquasi-ordered if $\left(\mathcal{C}, \leq_{\mathcal{C}}\right)$ is a wqo. The class $\mathcal{C}$ is infinitely well-quasi-ordered if, for any wqo $Q,\left(Q^{\mathcal{C}}, \leq_{Q^{\mathcal{C}}}\right)$ is a wqo.

Theorem 1 (Kruskal [8]). The class of finite trees is infinitely well-quasi-ordered with respect to $\vee$-embedding.

Corollary 1. The class $\mathcal{T}_{F}$ is infinitely well-quasi-ordered.

Lemma 2. Let $G$ be a finite series-parallel graph labeled by a quasi-order $Q$. There is a tree $T(G)$ of $\mathcal{T}_{F}$ such that $S P(T(G))$ is isomorphic to $G$.

Proof. We prove the result by induction on the number of vertices. To the one element series-parallel graph, we associate the one element tree labeled by 0 . Now consider a nontrivial decomposition $H+I$ (resp. $H \cup I$ ) of $G$. We choose $T(G)$ to be the tree whose maximum element $m$ is labeled by 1 (resp. by 2 ) and has two branches, one isomorphic to $T(H)$ and the other isomorphic to $T(I)$. This tree $T(G)$ is not necessarily unique; for example the complete graph on four elements has two different decomposition trees.

Corollary 2. The class of finite series-parallel graphs is infinitely well-quasiordered.

Proof. The class of finite series-parallel graphs is wqo; this is a direct consequence of Lemma 2 and Kruskal's theorem. Moreover one can associate to any $Q$-labeled finite series-parallel graph $(G, l)$ a $Q$-labeled tree $\left(T, l^{\prime}\right)$ of $\mathcal{T}_{F}$ such that $(G, l)=$ $S P\left(\left(T, l^{\prime}\right)\right)$. Since Kruskal's theorem holds for trees labeled by a wqo, the class of finite series-parallel graphs is infinitely wqo. 


\section{THE INTERVAL NOTION}

The decomposition of the finite $N$-free graphs is based on connectedness, but as we have seen, this argument does not hold in the infinite case. We need to consider another tool: the notion of an interval (Fraïssé [3]).

Definition 3. Let $R=(V, E)$ be a binary relation. An interval of $R$ is a subset $I \subseteq V$ such that, for any $x$ and $y$ of $I$, any element $z$ of $V \backslash I$ satisfies $(x, z) \in E \Leftrightarrow$ $(y, z) \in E$ and $(z, x) \in E \Leftrightarrow(z, y) \in E$. The singletons, $V$ and the empty set are intervals called trivial intervals.

It is easy to verify that the intersection and the union of two non-disjoint intervals are intervals. If $I$ is an interval of $R$ and $W$ is a subset of $V$, then $I \cap W$ is an interval of $R(W)$. If $I$ is an interval of $R$ and $Y$ is an interval of $R(I)$, then $Y$ is an interval of $R$.

A relation $R$ is indecomposable if the sole intervals of $R$ are trivial.

An interval partition of $R$ is a partition of $V$ into non-empty intervals. Let $P$ be an interval partition of $R$. The quotient of $R$ by $P$ is the relation $R / P=(P, E / P)$ in which $(I, J) \in E / P$ if there are $i \in I$ and $j \in J$ such that $(i, j) \in E$. We can also view $R / P$ as the induced subrelation $R(S)$ for any subset $S$ such that $|S \cap Y|=1$ for any $Y \in P$. It follows from this definition that if $I$ is an interval of $R / P$, then $\bigcup I$ is an interval of $R$.

Any nontrivial finite series-parallel graph is the union or the sum of two graphs; thus it has an interval partition into two nonempty intervals. Thus, no finite seriesparallel graph of cardinal strictly greater than two is indecomposable. In other words, when a finite indecomposable graph has more than two elements, it embeds $N$. D. Kelly has generalized this result to infinite graphs. I learned the following proof from P. Ille 6 .

Lemma 3 (Kelly [7]). An indecomposable graph with at least three vertices embeds $N$.

Proof. Let $a$ be a vertex of a graph $G=(V, E)$ such that no induced $N$ in $G$ contains a. Let $A^{+}=\{x \in V \backslash\{a\}:(x, a) \in E\}$ and $A^{-}=\{x \in V \backslash\{a\}:(x, a) \notin E\}$. Let $Y$ be a connected component of $G\left(A^{-}\right)$. Suppose, by way of contradiction, that $Y$ is not an interval of $G$. Let $b$ and $c$ be two elements of $Y$ and $d$ an element of $V \backslash Y$ such that $(b, d) \in E$ and $(c, d) \notin E$. Clearly, $d$ is different from $a$ and since $Y$ is a connected component of $G\left(A^{-}\right), d$ does not belong to $A^{-}$. Consequently $d$ belongs to $A^{+}$. Since $Y$ is a component of $G\left(A^{-}\right)$, there is a path $b=b_{0}, b_{1}, b_{2}, \ldots, b_{n}=c$ which connects $b$ to $c$ in $G\left(A^{-}\right)$. Since $(b, d) \in E$ and $(c, d) \notin E$, there exists $j$ such that $\left(b_{j}, d\right) \in E$ and $\left(b_{j+1}, d\right) \notin E$. This is a contradiction, because $G\left(\left\{a, b_{j}, b_{j+1}, d\right\}\right)$ is isomorphic to $N$. The graph $G$ is indecomposable, so the interval $Y$ is trivial, more precisely a singleton. Thus, the induced subgraph $G\left(A^{-}\right)$is an empty graph. By a similar argument, $G\left(A^{+}\right)$is a complete graph. Suppose, by way of contradiction, that there is another vertex $b$ of $G$ such that no induced $N$ in $G$ contains $b$. Without loss of generality, we suppose that $(a, b) \in E$ (otherwise we consider $G^{c}$ ). Let $z$ be a vertex of $G$ distinct from $a$ and $b$. If $z$ is adjacent to $a$, since $z$ and $b$ belong to the complete graph $G\left(A^{+}\right), b$ and $z$ are adjacent. Similarly, if $z$ is adjacent to $b$, it is also adjacent to $a$. Thus, $\{a, b\}$ is a nontrivial interval of $G$, a contradiction. 
A consequence of this lemma is that the indecomposable induced subgraphs of a series-parallel graph have one or two elements.

\section{Tree Decomposition of COUNTABle SERIES-PARALlEL GRAPhS}

We denote by $\mathcal{T}_{\mathcal{C}}$ the class of countable trees of $\mathcal{T}$. Our goal is to associate a tree of $\mathcal{T}$ to every series-parallel graph. This technique is similar to Kelly's notion of strong interval [7, but since we only need binary trees, the tree decomposition is easier to obtain.

Definition 4. Two sets $X$ and $Y$ improperly intersect each other if their intersection is $\emptyset, X$ or $Y$.

Let $R=(V, E)$ be a binary relation. A set $\mathcal{F}$ of non-empty intervals of $R$ is an interval family if it is maximal under inclusion and any two of its elements improperly intersect each other. From the definition, an interval family contains the set $V$ and the singletons. We order the set $\mathcal{F}$ by inclusion. It is routine to verify that $(\mathcal{F}, \subseteq)$ is a tree.

Lemma 4. Interval families are closed under increasing unions and non-empty intersections.

Proof. Let $\sigma$ be an increasing (with respect to inclusion) sequence of elements of $\mathcal{F}$ and $\Sigma$ be the union of $\sigma$. By the properties of intervals, $\Sigma$ is an interval of $R$. Moreover, $\Sigma$ intersects improperly any element of $\mathcal{F}$; thus it belongs to $\mathcal{F}$. The same proof holds for the intersection.

Corollary 3. If I is an element of $(\mathcal{F}, \subseteq)$ which has more than one branch, then each branch of $I$ has a maximum element.

Proof. Let $B$ be a branch of $I$ and $C$ a maximal subchain of $B$. From the lemma, $W=\bigcup C$ is an element of $\mathcal{F}$ which contains every element of $B$. Furthermore $I$ has more than one branch, so $W$ is distinct from $I$ and consequently belongs to $B$.

Corollary 4. The tree $(\mathcal{F}, \subseteq)$ is well-branched.

Proof. Let $A$ and $B$ be two elements of $\mathcal{F}$, and $C:=\{X \in \mathcal{F}: A \subseteq X, B \subseteq X\}$. The set $\bigcap C$ which is the supremum of $A$ and $B$ belongs to $\mathcal{F}$.

Theorem 2. Let $S=(V, E)$ be a countable series-parallel graph. For any interval family $\mathcal{F}$ of $S$, there is a canonical tree $T_{\mathcal{F}}$ of $\mathcal{T}_{\mathcal{C}}$ such that $S P\left(T_{\mathcal{F}}\right)=S$.

Proof. We prove first that the tree $(\mathcal{F}, \subseteq)$ is a binary tree. Let $I$ be an element of $\mathcal{F}$ such that $I$ has at least two branches in $(\mathcal{F}, \subseteq)$. By Corollary 3 each branch of $I$ has a maximum element. We denote by $P$ the set of maximum elements of the branches of $I$; in particular $P$ is a partition of $I$. Furthermore, any element of $P$ is an interval of $S$ (and thus an interval of $S(I)$ ), so $P$ is an interval partition of $S(I)$. Since $G$ is $N$-free, $G(I)$ and the quotient graph $S(I) / P$ are also $N$-free. Suppose, by way of contradiction, that $S(I) / P$ has more than two vertices. By Lemma 3, $S(I) / P$ has a nontrivial interval $I^{\prime}$. Thus $\bigcup I^{\prime}$ is an interval of $S$ which improperly intersects any interval of $\mathcal{F}$, a contradiction, since $\bigcup I^{\prime}$ does not belong to $\mathcal{F}$. Now, we label the elements of $(\mathcal{F}, \subseteq)$ by the 3 -element antichain $A_{3}$. The minimal elements of $(\mathcal{F}, \subseteq)$ are labeled by 0 . Let $I$ be an element of $\mathcal{F}$ which has two branches. If $S(I) / P$ is the empty graph with two elements, we label it by 2 ; 
if it is the complete graph with two elements, we label it by 1 . Now we remove all the elements of $(\mathcal{F}, \subseteq)$ with exactly one branch. Call this new tree $T_{\mathcal{F}}$. We claim that $T_{\mathcal{F}}$ satisfies the hypothesis of the lemma. The minimal elements of $T_{\mathcal{F}}$ are the singletons of $V$. Furthermore any nonminimal element of $T_{\mathcal{F}}$ is the supremum of two minimal elements; thus $T_{\mathcal{F}}$ is countable since there are countably many elements in $V$. Finally, $G=S P\left(T_{\mathcal{F}}\right)$ follows from the construction.

\section{BETTER-QUASI-ORDER OF COUNTABLE SERIES-PARALLEL GRAPHS}

To deal with countable structures, well-quasi-order is no longer an appropriate notion. Indeed, there exists a well-quasi-order $Q$ (for example Rado's counterexample [13]) such that the class of $Q$-labeled countable empty graphs is not a wqo class. Here, a stronger notion is necessary : the better-quasi-order of Nash-Williams. The following definition of better-quasi-order is included here to fix the ideas. It is of no use in the proofs. For a survey about this notion, see Milner [10].

Definition 5. A quasi-order $Q$ is a better-quasi-order (or bqo), if the class of countable ordinals labeled by $Q$ is a well-quasi-order. A class of relations $\mathcal{C}$ is infinitely better-quasi-ordered if, for any better-quasi-order $Q,\left(Q^{\mathcal{C}}, \leq_{Q^{\mathcal{C}}}\right)$ is a better-quasiorder.

We shall need the following properties of better-quasi-orders. Any finite order is a better-quasi-order. Any better-quasi-order is a well-quasi-order. Any extension of a better-quasi-order is a better-quasi-order. The set of initial sections of a betterquasi-order is better-quasi-ordered with respect to inclusion.

Theorem 3 (Laver 9]). The class of countable chains is infinitely better-quasiordered.

Theorem 4 (Corominas [1]). The class of countable trees is infinitely better-quasiordered with respect to $\vee$-embedding.

As in the finite case, if the series-parallel graph is labeled, the label is transferred to the minimal elements of its decomposition tree. By Corominas' theorem and the existence of a decomposition tree, the next theorem holds.

Theorem 5. The class of countable series-parallel graphs is infinitely better-quasiordered.

This bqo result does not hold any more for higher cardinalities. To show this, we use the chain $\mathcal{C}$ of Dushnik and Miller [2]. This chain is a dense subchain of the real chain, which has $2^{\aleph_{0}}$ elements and does not contain any strict copy of itself. It is then routine to prove that the class of chains on $2^{\aleph_{0}}$ elements is not wqo : consider a sequence $x_{0} \leq_{\mathcal{C}} x_{1} \leq_{\mathcal{C}} x_{2} \leq_{\mathcal{C}} \cdots \leq_{\mathcal{C}} x_{i} \leq_{\mathcal{C}} \ldots$ of elements of $\mathcal{C}$. From the definition of $\mathcal{C}$, the sequence $\mathcal{C}^{x_{0}}, \mathcal{C}^{x_{1}}, \mathcal{C}^{x_{2}}, \ldots \mathcal{C}^{x_{i}}, \ldots$ is a bad sequence. The same kind of argument is used to prove that the class of series-parallel graphs on $2^{\aleph_{0}}$ vertices is not bqo. For this purpose, we construct the series-parallel graph $\mathcal{S}$.

Definition 6. As before, we denote by $\mathcal{C}=(V, E)$ the chain of Dushnik and Miller. Since it is dense, we consider $l$, a labeling from $V$ into $\{0,1\}$ such that $l^{-1}(0)$ and $l^{-1}(1)$ are dense in $\mathcal{C}$. Now $\mathcal{S}=\left(V, E^{\prime}\right)$ where $(x, y) \in E^{\prime}$ if $x<_{\mathcal{C}} y$ and $l(y)=1$ or $y<_{\mathcal{C}} x$ and $l(x)=1$. One can easily check that this graph does not embed $N$.

Lemma 5. The nontrivial intervals of $\mathcal{S}$ are exactly the initial sections of $\mathcal{C}$. 
Proof. Clearly, the initial sections of $\mathcal{C}$ are intervals of $\mathcal{S}$. Let $I$ be a non-trivial interval of $\mathcal{S}$ and $x$ and $y$ two elements of $I$ such that $x<_{\mathcal{C}} y$. We prove that the initial section of $\mathcal{C}$ generated by $y$ is included in $I$. Let $z$ be an element of $\mathcal{S}$ such that $z<_{\mathcal{C}} y$. i) If $z<_{\mathcal{C}} x$, then $z$ is linked to exactly one vertex in $\{x, y\}$; thus $z$ belongs to $I$. ii) If $l(x)=l(y), l(z) \neq l(y)$ and $x<_{\mathcal{C}} z<_{\mathcal{C}} y$, then $z$ belongs to $I$ by the same argument. iii) More generally, if $l(x)=l(y)$, it follows from density and ii) that there exists a vertex $u \in I$ such that $l(u) \neq l(y)$ and $z<_{\mathcal{C}} u<_{\mathcal{C}} y$. Thus, we apply i) with $u$ in place of $x$. Thus $z$ belongs to $I$. iv) Finally, if $l(x) \neq l(y)$, by density and i), we choose $u \in I$ such that $u<_{\mathcal{C}} x$ and $l(u)=l(y)$. Thus we conclude using iii) with $u$ instead of $x$.

Corollary 5. The sequence of series-parallel graphs $\left\{\mathcal{S}^{i}\right\}_{i \in \omega}$ where

$$
\mathcal{S}^{i}=\mathcal{S}(V \cap] i, \rightarrow[)
$$

is strictly decreasing with respect to embedding.

Proof. Observe that if $f$ is an embedding and $I$ is an interval, then $f^{-1}(I)$ is an interval. Thus $\mathcal{S}$ does not contain any strict copy of itself, for this would imply that $\mathcal{S}$ had such a copy. Thus the sequence $\left\{\mathcal{S}^{i}\right\}_{i \in \omega}$ is strictly decreasing.

\section{THE SERIES-PARALLEL ORDERS}

The lemmas on series-parallel orders are similar to those concerning graphs; we simply state them without proof. We show a way to find a decomposition tree of a series-parallel order. We have to code here more information than for graphs, leading us to introduce the notion of structured tree.

Definition 7. The $N$ order is the order on vertex set $\{0,1,2,3\}$ and such that $0<1,2<1,2<3$. It is the only transitive orientation, up to isomorphism, of the $N$ graph. A series-parallel order is an order which does not embed $N$; for example, any tree is a series-parallel order.

Let $P=(V, E)$ and $P^{\prime}=(W, F)$ be two orders. The order $P \cup P^{\prime}=(V \cup W, E \cup F)$ is the union of $P$ and $P^{\prime}$. We define also $P+P^{\prime}=(V \cup W, E \cup F \cup(V \times W))$, the sum of $P$ and $P^{\prime}$.

A structured tree $(T, S)$ is a pair where $T=(V, E)$ is a well-branched tree and $S=\left\{\leq_{v}\right\}_{v \in V}$ is a set of orders such that, for any vertex $v \in V, \leq_{v}$ is a linear ordering on the set of branches of $v$. We denote by $\mathcal{S}_{L}$ the class of well-branched, structured, binary trees labeled by $A_{3}$ such that the minimal elements, and only these, are labeled 0 .

A structured tree $(T, S)$ is s-embedded into a structured tree $\left(T^{\prime}, S^{\prime}\right)$ if there is a $\vee$-embedding $f$ from $T$ into $T^{\prime}$ which preserves structure. That is, for any vertex $v$ of $T$ such that $B<{ }_{v} B^{\prime}$ where $B$ and $B^{\prime}$ are branches under $v$, we have $\bar{f}(B)<_{f}(v)$ $\bar{f}\left(B^{\prime}\right)$, where $\bar{f}(B)$ (resp. $\left.\bar{f}\left(B^{\prime}\right)\right)$ is the branch under $f(v)$ which contains $f(B)$ (resp. $f\left(B^{\prime}\right)$ ).

Let $(T, l, S)$ be an element of $\mathcal{S}_{L}$ (for short we denote it by $T$ ). The series-parallel order $S P(T)$ is the partial order on the vertex set $M$, the set of minimal elements of $T$, such that $x \leq_{S P(T)} y$ if and only if $l(x \vee y)=1$ and $B(x) \leq_{x \vee y} B(y)$ where $B(x)$ (resp. $B(y))$ is the branch of $x \vee y$ which contains $x$ (resp. $y$ ).

Theorem 6 (Kelly [7]). Any indecomposable order with at least three vertices embeds $N$. 
Proof. Similar to Lemma 3

Corollary 6. For any countable $N$-free order $P$ there is a countable tree $T$ of $\mathcal{S}_{L}$ such that $S P(T)$ is isomorphic to $P$.

Proof. Analogous to the proof of Theorem [2] The only difference occurs in the discussion on $S(I) / P$. Here, $S(I) / P$ is either the two-element antichain, in which case we label by 2 the node $I$, or the two-element chain, in which case we label by 1 the node $I$ and we structure the branches of $I$ according to the order of $S(I) / P$.

As for graphs, we translate the problem of bqo of countable series-parallel orders into a problem of bqo of a class of trees. Indeed, we just have to prove now that the class $\mathcal{S}$ of countable trees of $\mathcal{S}_{L}$ is a bqo. The main problem underlying the proof of bqo of $\mathcal{S}$ is to find a construction of this class using operations which 'behave well' with respect to bqo, so that the class $\mathcal{S}$ is an algebra containing a bqo basis and is closed under these operations. For the precise definitions, see Pouzet [12].

In the case of chains, the simpler operation is the sum, as defined previously. But, obviously, this operation is not enough to construct the class of countable chains. For example, there are some chains $C$ such that, for any partition $C=C_{1}+C_{2}$, the chain $C$ is embedded into $C_{1}$ or $C_{2}$. The idea is to use these particular chains to construct the whole class. In Corominas' proof, a special class of trees plays the same role.

The following proof is an extension of Corominas' one to structured trees. Since our trees are binary, we prove that the class $\mathcal{S}$ can be constructed using two operations, instead of four in the original proof.

\section{Tree Partitions AND TRILABELING}

In what follows, $Q$ is a quasi-order.

Definition 8. Let $T=(V, E)$ be a tree of $\mathcal{S}$ and $\mathcal{C}$ a class of $Q$-labeled trees of $\mathcal{S}$. Note that the canonical embedding between elements of $\mathcal{S}$ is the $A_{3}-\vee$-s-embedding. By embedding between trees of $\mathcal{S}$, we always mean this canonical embedding.

A trilabeling of $T$ is a triple of mappings $\left(l_{1}, l_{2}, l_{3}\right)$ such that:

i) The domain of $l_{1}$ is a subset of vertices of $T$ which have exactly one branch. The range of $l_{1}$ is $\mathcal{C} \times\{0,1\}$.

ii) The domain of $l_{2}$ is a subset of minimal vertices of $T$. The range of $l_{2}$ is $\mathcal{C} \times \mathcal{C}$.

iii) The domain of $l_{3}$ is a finite or countable subset of maximal chains of $T$ without minimal elements. The range of $l_{3}$ is $\mathcal{C}$.

The tree $T$ together with $\left(l_{1}, l_{2}, l_{3}\right)$ is a trilabeled tree; $\mathcal{C}$ is the range of the trilabel. Let $\left(T^{\prime},\left(l_{1}^{\prime}, l_{2}^{\prime}, l_{3}^{\prime}\right)\right)$ be a trilabeled tree. A $t$-embedding $f$ from $\left(T,\left(l_{1}, l_{2}, l_{3}\right)\right)$ into $\left(T^{\prime},\left(l_{1}^{\prime}, l_{2}^{\prime}, l_{3}^{\prime}\right)\right)$ is an embedding $f$ from $T$ into $T^{\prime}$ such that for any vertex $x \in T$, $l_{1}(x) \leq l_{1}^{\prime}(f(x))$ and $l_{2}(x) \leq l_{2}^{\prime}(f(x))$ and for any maximal chain $C$ which belongs to the domain of $l_{3}$ there is a maximal chain $C^{\prime}$ of $T^{\prime}$ which contains $f(C)$ and is such that $l_{3}(C) \leq l_{3}^{\prime}\left(C^{\prime}\right)$.

For any vertex $x$ of $T$, the trilabeled tree $\left(T_{x},\left(l_{1}, l_{2}, l_{3}\right)\right)$ is the tree $T_{x}$ together with the restrictions of $l_{1}$ and $l_{2}$ to the elements less than $x$ and the restriction of $l_{3}$ to the maximal chains which contain $x$.

The realization of $\left(T,\left(l_{1}, l_{2}, l_{3}\right)\right)$, denoted by $\left[T,\left(l_{1}, l_{2}, l_{3}\right)\right]$, is the $Q$-labeled tree of $\mathcal{S}$ constructed from $T$ in the following way: 
i) Whenever $x$ is a vertex of $T$ such that $l_{1}(x)=\left(T^{\prime}, 0\right)\left(\right.$ resp. $l_{1}(x)=\left(T^{\prime}, 1\right)$ ), add the branch $T^{\prime}$ under $x$ with the structure $T^{\prime}<_{x} B$ (resp. $B<_{x} T^{\prime}$ ) where $B$ is the unique branch of $x$ in $T$.

ii) If $x$ is a minimal vertex of $T$ such that $l_{2}(x)=\left(T^{\prime}, T^{\prime \prime}\right)$, add the branches $T^{\prime}$ and $T^{\prime \prime}$ under $x$ with the structure $T^{\prime}<_{x} T^{\prime \prime}$.

iii) If $l_{3}(C)=T^{\prime}$, add the tree $T^{\prime}$ under the chain $C$.

It is easy to verify that if $\left(T,\left(l_{1}, l_{2}, l_{3}\right)\right)$ is $t$-embedded into $\left(T^{\prime},\left(l_{1}^{\prime}, l_{2}^{\prime}, l_{3}^{\prime}\right)\right)$, then $\left[T,\left(l_{1}, l_{2}, l_{3}\right)\right]$ is embedded into $\left[T^{\prime},\left(l_{1}^{\prime}, l_{2}^{\prime}, l_{3}^{\prime}\right)\right]$.

Lemma 6. Let $T=(V, E)$ be a $Q$-labeled tree of $\mathcal{S}$ and $\{I, F\}$ a partition of $V$ such that $I$ is an initial section of $T$ and $F$ a final one. We denote by $\mathcal{C}$ the set of components of the forest $T(I)$. Then there exists a trilabeling $\left(l_{1}, l_{2}, l_{3}\right)$ of $T(F)$ whose range is $\mathcal{C}$ such that $T$ is isomorphic to $\left[T(F),\left(l_{1}, l_{2}, l_{3}\right)\right]$.

Proof. Let $B$ be a nonempty component of $T(I)$. The final section generated by $B$ in $T$ intersects $F$ following a chain $C$. If $C$ has a minimum $x$, we add $B$ under $x$ using $l_{1}$ or $l_{2}$, depending on the number of branches of $x$ included in $I$. If $C$ has no minimum, in order to add $B$ under $C$ using $l_{3}$, we have to check that $C$ is maximal under inclusion in $T(F)$. Suppose, by way of contradiction, that there is a vertex $x \in F$ such that $x<_{T} C$. Pick a vertex $z$ of $B$. The supremum of $z$ and $y$ does not exist, a contradiction.

We refer to the trilabeling of this lemma as the trilabeling associated to the partition $\{I, F\}$.

\section{Strongly indecomposable trees}

Definition 9. Let $T=(V, E)$ be a tree of $\mathcal{S}$ labeled by $Q$.

The tree $T$ is strongly indecomposable if, for any vertex $x$ of $T$, the subtree $T_{x}$ embeds $T$. The empty tree and the singleton tree are the sole strongly indecomposable finite trees.

Let $Y$ be a subset of $V$ such that $T(Y)$ is a chain and let $l_{Y}$ be a mapping from $Y$ into $A_{3} \times A_{2}$. We call $\left(T(Y), l_{Y}\right)$ a structured chain of $T$ if $l_{Y}$ is defined in the following way: if $y$ is not the minimum of $T(Y)$, then $l_{Y}(y)=(i, j)$ where $i$ is the number of branches of $y$ in $T$ and $j$ is equal to 1 if and only if $y$ has two branches $B_{1}$ and $B_{2}$ in $T, B_{1}<_{y} B_{2}$ and $Y \cap B_{2} \neq \emptyset$ (and thus $Y \cap B_{1}=\emptyset$ ). If $y$ is the minimum of $T(Y)$, then $l_{Y}(y)=(0,0)$.

We denote by $B(T)$ the initial section in the class of $\{0,1,2\} \times\{0,1\}$-labeled countable chains, generated by the set of structured chains of $T$. Hence $(C, l) \in$ $B(T)$ if and only if there exists a structured chain $\left(T(Y), l_{Y}\right)$ of $T$ which embeds $(C, l)$.

The tree $T$ is stable if $B(T)=B\left(T_{x}\right)$ for any vertex $x$ of $T$.

Let $\left(T,\left(l_{1}, l_{2}, l_{3}\right)\right)$ be a trilabeled tree of $\mathcal{S}$ whose range is in $\mathcal{C}$, a subclass of $\mathcal{S}$. The tree is strongly indecomposable if, for any vertex $x$ of $T,\left(T,\left(l_{1}, l_{2}, l_{3}\right)\right)$ is $t$-embedded into $\left(T_{x},\left(l_{1}, l_{2}, l_{3}\right)\right)$.

The typical example of a strongly indecomposable tree is the binary tree of height $\omega\left(T^{x}\right.$ is finite for any $x$ and any vertex has two branches). One can easily check that any strongly indecomposable tree which is not a chain embeds this binary tree.

Lemma 7. Let $T=(V, E)$ and $T^{\prime}=\left(V^{\prime}, E^{\prime}\right)$ be two trees of $\mathcal{S}$. If $B(T) \subseteq B\left(T^{\prime}\right)$ and $T^{\prime}$ is stable, then $T^{\prime}$ embeds $T$. 
Proof. The set of vertices of a countable tree can be covered by a countable set of chains. Let $V=\bigcup\left\{V_{i}\right\}_{i \in \omega}$ where $T\left(V_{i}\right)$ is a maximal chain of $T$ with respect to inclusion. We construct by induction the embedding of $T$ into $T^{\prime}$. Since $B(T) \subseteq$ $B\left(T^{\prime}\right)$, there exists an embedding $f_{0}$ from $\left(T\left(V_{0}\right), l_{V_{0}}\right)$ into a structured chain of $T^{\prime}$; thus $\left(T\left(V_{0}\right), l_{V_{0}}\right)$ is isomorphic to $\left(T^{\prime}\left(f_{0}\left(V_{0}\right)\right), l_{f_{0}\left(V_{0}\right)}\right)$.

Now, we assume that we have already defined the embedding $f_{n}$ from $T_{n}=$ $T\left(\bigcup\left\{V_{i}\right\}_{i<n}\right)$ into $T^{\prime}$ in such a way that the structured chain $\left(T\left(V_{i}\right), l_{V_{i}}\right)$ is isomorphic to $\left(T^{\prime}\left(f_{n}\left(V_{i}\right)\right), l_{f_{n}\left(V_{i}\right)}\right)$ for any $0 \leq i<n$. We want to extend this embedding to $V_{n}$. There is $j<n$ such that $V_{n} \cap\left(\bigcup\left\{V_{i}\right\}_{i<n}\right)=V_{j} \cap V_{n}$. If $V_{j}=V_{n}$, there is nothing to prove. If $V_{j} \neq V_{n}$, since $T$ is well-branched and these chains are maximal, there is one vertex $x$ of $V_{j}$ such that $\left(V_{j} \cap V_{n}\right)=T^{x}$. Now two cases may occur : $l_{V_{j}}(x)=(2,0)$ or $l_{V_{j}}(x)=(2,1)$.

Since the proofs are similar, we only treat the first case. From the induction hypothesis, it follows that $l_{f_{n}\left(V_{j}\right)}\left(f_{n}(x)\right)=l_{V_{j}}(x)=(2,0)$; thus $f_{n}(x)$ has two branches $B_{1}^{\prime} \leq_{f_{n}(x)} B_{2}^{\prime}$ and $f_{n}\left(V_{j}\right) \cap B_{1}^{\prime} \neq \emptyset$. Since $x$ is the meeting vertex of $V_{j}$ and $V_{n}$, then $l_{V_{n}}(x)=(2,1)$. Now, we just have to embed $V_{n} \backslash T^{x}$ into $B_{2}^{\prime}$. Let $x^{\prime}$ be an element of $B_{2}^{\prime}$, by the hypothesis $B(T) \subseteq B\left(T^{\prime}\right)=B\left(T_{x^{\prime}}^{\prime}\right)$; thus we can extend $f_{n}$ to $V_{n}$.

Corollary 7. Let $T$ be a stable tree of $\mathcal{S}$. Then $T$ is strongly indecomposable.

Corollary 8. Let $T$ be a tree of $\mathcal{S}$. There is a vertex $x$ of $T$ such that $T_{x}$ is stable.

Proof. We consider $\left\{B\left(T_{x}\right), x\right.$ is a vertex of $\left.T\right\}$. This is a set of initial sections of the class of $A_{3} \times A_{2}$-labeled chains, which is bqo by virtue of Laver's theorem. Thus, the set $\left\{B\left(T_{x}\right), x\right.$ is a vertex of $\left.T\right\}$ is bqo with respect to inclusion and consequently has minimal elements. Let $x$ be a vertex such that $B\left(T_{x}\right)$ is minimal. Clearly $T_{x}$ is stable.

Corollary 9. The class of strongly indecomposable trees of $\mathcal{S}$ is better-quasi-ordered with respect to embedding.

Proof. Given a strongly indecomposable tree $T$ of $\mathcal{S}$, there exists, by Corollary 8 a stable subtree $T_{x}$ of $T$. Since $T$ is strongly indecomposable, $T_{x}$ is equimorphic to $T$. Now, we just have to prove that the class of stable trees of $\mathcal{S}$ is bqo. This is a consequence of Lemma 7 and Laver's result.

Lemma 8. Let $\left(T,\left(l_{1}, l_{2}, l_{3}\right)\right)$ be a trilabeled tree of $\mathcal{S}$ such that the range of the trilabeling is a bqo. Then there exists a vertex $x$ of $T$ such that $\left(T_{x},\left(l_{1}, l_{2}, l_{3}\right)\right)$ is strongly indecomposable.

Proof. Analogous to the proof of Corollary 8 .

\section{Canonical trees and the Reduction process}

We present in this section a construction of a subclass of $\mathcal{S}$, the canonical trees. This construction is based on the notion of indecomposable trees.

Definition 10. Let $\mathcal{B}$ be a subclass of $\mathcal{S}$. We define two operations on $\mathcal{B}$ :

i) The tree operation associates the tree $\left[T,\left(l_{1}, l_{2}, l_{3}\right)\right]$ to any strongly indecomposable trilabeled tree $\left(T,\left(l_{1}, l_{2}, l_{3}\right)\right)$ with range contained in $\mathcal{B}$.

ii) The chain operation associates the tree $\left[C,\left(l_{1}, l_{2}, l_{3}\right)\right]$ to any trilabeled chain $\left(C,\left(l_{1}, l_{2}, l_{3}\right)\right)$ with range contained in $\mathcal{B}$. 
The minimum subclass of $\mathcal{S}$ which contains the empty tree and is closed with respect to the two previous operations is called the class of canonical trees.

Theorem 7 (Corominas [1]). The class of canonical trees is infinitely bqo.

The proof of this theorem uses ordinal algebras (Pouzet [12]). The structure of the trees only adds another labeling to Corominas' original proof. Now we will prove that this class is indeed equal to $\mathcal{S}$. The key idea is to reduce the trees of $\mathcal{S}$ by iterations of Lemma 8 . This gives a process of reduction of the trees which terminates after countably many steps.

Definition 11. Let $T=(V, E)$ be a tree of $\mathcal{S}$.

We define by induction the $\alpha$-partition of $V$ into two subsets $\left\{I^{\alpha}, F^{\alpha}\right\}$ such that $I^{\alpha}$ is an initial section and $F^{\alpha}$ is a final section.

i) The 0-partition of $V$ is $\{\emptyset, V\}$, denoted by $\left\{I^{0}, F^{0}\right\}$.

ii) If $\alpha$ is a limit ordinal, the $\alpha$-partition of $V$ is $\left\{I^{\alpha}, F^{\alpha}\right\}$ in which $F^{\alpha}=$ $\bigcap\left\{F^{\beta}\right\}_{\beta<\alpha}$ and thus $I^{\alpha}=V \backslash F^{\alpha}$.

iii) If $\alpha=\beta+1$, following Lemma [6 we can associate a tree $\left(T\left(F^{\beta}\right),\left(l_{1}, l_{2}, l_{3}\right)\right)$ to the partition $\left\{I^{\beta}, F^{\beta}\right\}$ such that $\left(T\left(F^{\beta}\right),\left(l_{1}, l_{2}, l_{3}\right)\right)=T$. Now, a vertex $x$ of $F^{\beta}$ still belongs to $F^{\alpha}$ if and only if $\left(T\left(F^{\beta}\right)_{x},\left(l_{1}, l_{2}, l_{3}\right)\right)$ is not strongly indecomposable. Note that if $\left(T\left(F^{\beta}\right)_{x},\left(l_{1}, l_{2}, l_{3}\right)\right)$ is strongly indecomposable, then $\left(T\left(F^{\beta}\right)_{y},\left(l_{1}, l_{2}, l_{3}\right)\right)$ is also strongly indecomposable for any vertex $y$ of $F^{\beta}$ such that $y \leq x$. Thus, $F^{\alpha}$ is a final section.

We call the degree of $T$, denoted by $d(T)$, the least ordinal $\alpha$, when it exists, such that the final section $F^{\alpha}$ is empty.

\section{BQO OF COUNTABLE SERIES-PARALlEL ORDERS}

We now have all the definitions required to prove the bqo property of the class $\mathcal{S}$. On the one hand, we know that the class of canonical trees is bqo and on the other hand we have a reduction process for the elements of $\mathcal{S}$ in order to prove that all of them are canonical.

Lemma 9. If $T$ is a tree of $\mathcal{S}$ such that $T_{x}$ is canonical for any vertex $x$, then $T$ is canonical.

Proof. For any vertex $x$ of a canonical tree, the branches of $x$ are canonical trees. Let $C$ be a maximal chain of $T$. We can write $T=\left[C,\left(l_{1}, l_{2}, l_{3}\right)\right]$, where the domain of $l_{3}$ is empty and the ranges of $l_{1}$ and $l_{2}$ are sets of canonical trees. Consequently, $T$ is canonical.

Lemma 10. Any tree of $\mathcal{S}$ which has a countable degree is canonical.

Proof. By induction on the degree. Let $T$ be a tree such that $d(T)=\alpha$.

i) If $d(T)=1$, then $T$ is strongly indecomposable. Thus $T=[T,(\emptyset, \emptyset, \emptyset)]$ is canonical.

ii) If $\alpha$ is a limit ordinal, for any vertex $x$ there is an ordinal $\beta<\alpha$ such that $x \in F^{\beta}$ and $x \notin F^{\beta+1}$. By the induction hypothesis, $T_{x}$ is canonical for any $x$. It follows from Lemma 9 that the tree $T$ is also canonical.

iii) If $\alpha=\beta+1$, the partition $\left\{I^{\beta}, F^{\beta}\right\}$ is such that $\left(T\left(F^{\beta}\right)_{x},\left(l_{1}, l_{2}, l_{3}\right)\right)$ is strongly indecomposable for any vertex $x$ in $F^{\beta}$. By the induction hypothesis the labels are canonical trees; hence $\left[T\left(F^{\beta}\right)_{x},\left(l_{1}, l_{2}, l_{3}\right)\right]=T_{x}$ is canonical for any $x$ of $F^{\beta}$. Now, if $x$ belongs to $I^{\beta}$, then $d\left(T_{x}\right) \leq \beta$ and consequently $T_{x}$ is canonical. We again apply Lemma 9 to prove that $T$ is canonical. 
Lemma 11. Any tree of $\mathcal{S}$ has a countable degree.

Proof. Let $\alpha$ be a countable ordinal such that $F^{\alpha}$ is not empty. In $\left(T\left(F^{\alpha}\right),\left(l_{1}, l_{2}, l_{3}\right)\right)$, the labels are canonical; hence they are bqo. By Lemma 8 , there is a vertex $x$ of $F^{\alpha}$ such that $\left.\left(T\left(F^{\alpha}\right)_{x},\left(l_{1}, l_{2}, l_{3}\right)\right)\right)$ is strongly indecomposable. Thus $x$ is not an element of $F^{\alpha+1}$, so the sequence $\left\{F^{\alpha}\right\}_{\alpha<\omega_{1}}$ is strictly decreasing. Since the trees of $\mathcal{S}$ are countable, they have countable degree.

We have proved that $\mathcal{S}$ is exactly the class of canonical trees. So we have shown that :

Theorem 8. The class $\mathcal{S}$ is infinitely better-quasi-ordered.

Corollary 10. The class of countable trees of $\mathcal{S}_{L}$ is infinitely better-quasi-ordered.

And finally:

Theorem 9. The class of countable series-parallel orders is infinitely better-quasiordered.

\section{Compactness of WQO AND Related Questions}

If a class of infinite relations is infinitely bqo, the property holds in particular for any relation $R$ of this class. That is, for any bqo $Q$, the class of $Q$-labelings of $R$ is bqo. This remark suggests that $R$ embeds many distinct copies of itself. This is indeed the case, as we can see in the following result, expressed in terms of series-parallel orders:

Lemma 12. Let $S=(V, E)$ be a countable series-parallel order. Then $S$ embeds at least one strict copy of itself.

Proof. We label $S$ by $A_{2}=\{0,1\}$, the two element antichain. We consider a sequence $\left(S, l_{0}\right),\left(S, l_{1}\right), \ldots,\left(S, l_{i}\right), \ldots$ where $l_{i}$ is a labeling of $S$ such that exactly $i$ elements of $V$ are labeled by 0 . Since one can find $i<j$ such that $\left(S, l_{i}\right)$ is embedded into $\left(S, l_{j}\right)$, this embedding avoids at least $j-i$ vertices labeled by 0 . In fact, using $A_{2}$-labelings, we can prove with the aid of Bernstein's lemma that there exists more than a countable set of copies of $S$.

Theorem 10 (Thomassé [14]). Let $S$ be a countable series-parallel order. Then $S$ contains $2^{\omega}$ copies of itself intersecting one another in finite sets.

Proof. We just give a sketch of the proof: the set of copies of $S$ is a Borel subset of the power set of $S$ endowed with the Tychonoff product topology. Indeed this is true for any relation and can be proved using the Scott rank of $S$. Moreover, if the class of $A_{3}$-labelings of a countable relation is a well-quasi-order, its set of copies is a comeager set. This property implies the theorem.

Let us introduce now two challenging conjectures concerning wqo and bqo of classes of structures. First note that all the results in the finite case extend to the countable case: Higman's wqo theorem for finite chains extends to Laver's theorem; Kruskal's theorem extends to that of Corominas, and finally the class of countable series-parallel orders is bqo. There is no example of an infinitely wqo class of finite structures which is not known to be infinitely bqo in the countable case. The question of labeling is also interesting. Do we really need these infinitely wqo or bqo properties? 
Definition 12. A class of finite relations $\mathcal{A}$ is an age if any two relations $R$ and $R^{\prime}$ of $\mathcal{A}$ are embedded in some $R^{\prime \prime} \in \mathcal{A}$ (the joint embedding property) and for any $R \in \mathcal{A}$, any relation embedded in $R$ belongs to $\mathcal{A}$. Clearly, the class of finite relations embedded in a given relation is an age. The class of countable relations which have a given age $\mathcal{A}$ is denoted by $C(\mathcal{A})$. We recall that $A_{2}$ denotes the 2-element antichain.

Conjecture 1 (Fraïssé, unpublished). If the class of $A_{2}$-labelings of $\mathcal{A}$ is wqo, then $\mathcal{A}$ is infinitely wqo.

Conjecture 2 (Pouzet [12]). If $\mathcal{A}$ is infinitely wqo, then $C(\mathcal{A})$ is infinitely bqo.

The first natural question on compactness of wqo does not hold. It can be the case that $\mathcal{A}$ is wqo and $C(\mathcal{A})$ is not. Such an example was found by Pouzet using pseudo-periodic sequences. For example, the Thue-Morse sequence $0110100110010110 \ldots$ seen as an infinite path $P$ labeled by $A_{2}$ has the following properties : it does not embed strictly in itself (otherwise the sequence would be periodic); hence the set of its final sections is a strictly decreasing sequence with respect to embedding. Moreover, for any integer $n$ there is an $m$ such that any finite subpath of length $n$ is embedded into any finite subpath of length $m$. This last property implies that the age of this labeled path is wqo.

\section{ACKNOWLEDGEMENT}

I gratefully thank M. Pouzet who introduced me this problem and J.A. Bondy for his help during the preparation of this paper.

\section{REFERENCES}

[1] E. Corominas, On better-quasi-ordering countable trees, in Proceedings of the Conference on Ordered Sets and their Applications (L'Arbresle, July 1982), (M. Pouzet and D. Richard, eds.), Discrete Math., 53 (1984), 35-53. MR 86k:06001

[2] B. Dushnik and E.W. Miller, Concerning similarity transformations of linearly ordered sets, Bull. Amer. Math. Soc., 46 (1940), 322-326. MR 1:318g

[3] R. Fraïssé, L'intervalle en théorie des relations, ses généralisations, filtre intervallaire et clôture d'une relation, in Orders, Description and Roles, (M. Pouzet and D. Richard, eds.), Annals of Discrete Math., 23 North-Holland, (1984), 313-341. MR 85k:06001

[4] T. Gallai, Transitiv Orientierbare Graphen, Acta Math. Acad. Sci. Hungar., 18 (1967), 25-66. MR 36:5026

[5] G. Higman, Ordering by divisibility in abstract algebras, Proc. London Math. Soc., 2 (1952), 326-336. MR 14:238e

[6] P. Ille, Private communication.

[7] D. Kelly, Comparability graphs, in Graphs and Orders, (I. Rival ed.), D. Reidel Publishing Company, (1985), 3-40. MR 87g:05190

[8] J. B. Kruskal, Well-quasi-ordering, the tree theorem and Vázsonyi's conjecture, Trans. Amer. Math. Soc., 95 (1960), 210-225. MR 22:2566

[9] R. Laver, On Fraïssé's order type conjecture, Ann. of Math., 93 (1971), 89-111. [MR 43:4731

[10] E.C. Milner, Basic wqo and bqo theory, in Graphs and Orders, (I. Rival ed.), D. Reidel Publishing Company, (1985), 487-502. MR 87h:04004

[11] C.St.J.A. Nash-Williams, On better-quasi-ordering transfinite sequences, Proc. Camb. Phil. Soc., 64 (1968), 273-290. MR 36:5001

[12] M. Pouzet, Applications of well-quasi-ordering and better-quasi-ordering, in Graphs and Orders, (I. Rival ed.), D. Reidel Publishing Company, (1985), 503-519. MR 87g:06014 
[13] R. Rado, Partial well-ordering of sets of vectors, Mathematika, 1 (1954), 89-95. MR 16:576b

[14] S. Thomassé, Thèse de doctorat, Lyon (1995).

Laboratoire LMd, UFR de Mathématiques, Université Claude Bernard 43, Boulevard du 11 novembre 1918, 69622 Villeurbanne Cedex, France

E-mail address: thomasse@jonas.univ-lyon1.fr 